\title{
Phase II study of sequential high-dose methotrexate and fluorouracil combined with doxorubicin as a neoadjuvant chemotherapy for scirrhous gastric cancer
}

\author{
Shinichiro Takahashi ${ }^{1}$, Taira Kinoshita ${ }^{1}$, Masaru Konishi ${ }^{1}$, Toshio Nakagouri ${ }^{1}$, Kazuto Inoue ${ }^{1}$, \\ Masato Ono ${ }^{1}$, Masanori Sugitou ${ }^{1}$, Atsushi Ohtsu ${ }^{2}$, Narikazu Boku ${ }^{2}$, and Shigeaki Yoshida ${ }^{2}$ \\ ${ }^{1}$ Department of Surgery, National Cancer Center Hospital East, 6-5-1 Kashiwanoha, Kashiwa, Chiba 277-8577, Japan \\ ${ }^{2}$ Division of Digestive Endoscopy/Gastrointestinal Oncology, National Cancer Center Hospital East, Chiba, Japan
}

\begin{abstract}
Background. The prognosis of scirrhous gastric cancer remains poor when it is treated with surgical resection alone or chemotherapy alone. A phase II study of sequential high-dose methotrexate and fluorouracil, combined with doxorubicin, as a neoadjuvant chemotherapy was conducted in an attempt to evaluate the efficacy of this regimen in improving the survival of patients with scirrhous gastric cancer.

Methods. Patients were eligible if they had potentially resectable scirrhous gastric cancer with adequate organ functions and no prior treatment. The treatment schedule consisted of methotrexate $\left(1 \mathrm{~g} / \mathrm{m}^{2}\right.$, day 1$)$ fluorouracil $\left(1.5 \mathrm{~g} / \mathrm{m}^{2}\right.$, day 1$)$, leucovorin (15 mg/m², days $2-4)$, and doxorubicin $\left(30 \mathrm{mg} / \mathrm{m}^{2}\right.$, day 15), repeated at a 28-day interval, and followed by radical surgery.

Results. A total of 20 eligible patients were registered. Objective responses in the neoadjuvant chemotherapy segment were observed in 3 of the $20(15 \%)$ patients. No complete remission was observed. The neoadjuvant chemotherapy was associated with grade 3 or 4 neutropenia in 14 of the $20(70 \%)$ patients. The median time from the initial therapy to the operative day was 82 days. Thirteen of the $20(65 \%)$ patients underwent curative resection. No treatment-related deaths occurred. However, the 2-year survival rate in this treatment program $(25 \%)$ did not show any superiority over that in historical controls.

Conclusions. Sequential high-dose methotrexate and fluorouracil, combined, with doxorubicin, as a neoadjuvant chemotherapy for scirrhous gastric cancer did not improve the survival rate in spite of improving the curative resection rate.
\end{abstract}

Key words Scirrhous gastric cancer $\cdot$ FAMTX $\cdot$ Neoadjuvant chemotherapy

Offprint requests to: T. Kinoshita

Received: August 2, 2001 / Accepted: September 27, 2001
Introduction

The results of treatment for gastric cancer overall have gradually been improved by the detection of gastric cancers at earlier stages, better management in the postoperative period, and the development of effective chemotherapeutic regimens for advanced gastric cancers. However, the prognosis of scirrhous gastric cancer still remains far worse than that of other gastric cancers. Results from the treatment for gastric cancer patients at the National Cancer Center Hospital (NCCH) (19801990, Tokyo) show that the 5-year survival rate of patients with scirrhous gastric cancer treated with surgery is only $9 \%(25 / 265)$ : that of curatively resected patients is $19 \%$, and that of noncuratively resected scirrhous gastric cancer is only $3 \%$. These results for scirrhous gastric cancer have remained unchanged in the past decade. Currently, cure for patients with scirrhous gastric cancer is not assured, even in patients who undergo complete surgical resection with tumor-free margins followed by adjuvant systemic chemotherapy. In order to improve the prognosis for scirrhous gastric cancer, new therapeutic strategies, including neoadjuvant chemotherapy with effective drugs, are needed.

In Japan, several phase II studies of combination chemotherapy for advanced gastric cancer have been conducted. Combinations have included such agents as doxifluridine (5'DFUR) + cisplatin (CDDP) [1], etoposide + Adriamycin (doxorubicin) + CDDP (EAP) [2], and 5-fluorouracil + CDDP (FP) [3]. Although their survival benefits compared with singleagent therapy have not been elucidated, these combination regimens have yielded relatively high response rates, of $35 \%, 50 \%$, and $33 \%$, respectively. EAP provided the highest response rate of these three regimens. However, the response rate for EAP in patients with scirrhous gastric cancer was only $17 \%$, and the toxicity of this regimen was substantial, including a treatment-related mortality of approximately $10 \%[4,5]$. 
The remaining two regimens also showed little activity for scirrhous cancers.

In Europe, Klein et al. [6] and Wils et al. [7] reported a response rate of $63 \%$ with the sequential administration of high-dose methotrexate (MTX) with leucovorin rescue and 5-fluorouracil (5-FU) combined with Adriamycin (FAMTX). The results of a phase III multicenter trial by the European Organization for Treatment of Cancer (EORTC) demonstrated that the FAMTX regimen yielded a superior response rate and survival with less hematologic toxicity, compared with 5-FU + Adriamycin + mitomyciu C (FAM) [8]. Based on these results, FAMTX appeared to have become a standard regimen for gastric cancer in the United States and Europe during the 1990s [9-13], and a pilot study to evaluate the therapeutic effect of FAMTX for patients with unresectable scirrhous gastric cancer was conducted at our institution. Four of 12 patients (33\%) treated with FAMTX showed a partial response, which was the highest response rate shown for scirrhous gastric cancer among the many regimens attempted in our institution. Considering these results, we conducted a phase II study of neoadjuvant chemotherapy with FAMTX, followed by surgery, in patients with potentially resectable scirrhous gastric cancer.

\section{Subjects and methods}

\section{Patient eligibility}

Patient eligibility required the fulfilment of the following criteria: gastric adenocarcinomas with histological proof of a potentially resectable scirrhous type; no prior treatment; age, 70 years or younger; and Eastern Cooperative Oncology Group (ECOG) performance status of 0 or 1 . Patients also had to have adequate organ functions (creatinine clearance, $\geqq 60 \mathrm{ml} / \mathrm{min}$; blood urea creatinine, $\leqq 1.5 \mathrm{mg} / \mathrm{dl}$; GOT and GPT, $\leqq 100 \mathrm{U}$; total bilirubin, $\leqq 2.0 \mathrm{mg} / \mathrm{dl}$; leukocyte, $\geqq 4000 / \mathrm{mm}^{3} ;$ and thrombocytes, $\left.\geqq 100000 / \mathrm{mm}^{3}\right)$. Diagnostic and staging procedures included physical examination, barium gastrography, endoscopy, chest X-ray, and abdominal computed tomography scan. If possible, preoperative peritoneal washing cytology of the Douglas pouch was performed, under local anesthesia, in order to evaluate any peritoneal dissemination that was not detectable by medical imaging. This study was approved by the Institutional Review Board, and written informed consent was obtained from all patients.

\section{Treatment schedule}

Chemotherapy consisted of MTX, $1.0 \mathrm{~g} / \mathrm{m}^{2}$, followed by 5 -FU at $1.5 \mathrm{~g} / \mathrm{m}^{2}$ within a 1 -h interval; both drugs were administered as a bolus injection on day 1 . One liter of isotonic sodium bicarbonate $\left(\mathrm{NaHCO}^{3} ; 1.4 \%\right)$ was administered before MTX, and urine $\mathrm{pH}$ had to be maintained at 7.0 or more. After the administration of MTX, 21 of $\mathrm{NaHCO}^{3}, 1.4 \%$, was continuously infused over $24 \mathrm{~h}$. The plasma MTX level was monitored at 24 and $48 \mathrm{~h}$ after the administration of MTX, and leucovorin rescue was started from $24 \mathrm{~h}$ after MTX, at a dose of $15 \mathrm{mg} / \mathrm{m}^{2}$, given orally or intravenously, every $6 \mathrm{~h}$ for $48 \mathrm{~h}$. If the plasma MTX level after $48 \mathrm{~h}$ was higher than $1 \times 10^{-6} \mathrm{~mol} / \mathrm{l}$, the rescue had to be continued. On day 15 , Adriamycin, $30 \mathrm{mg} / \mathrm{m}^{2}$, was administered in a 1-h infusion. This schedule was carried out twice, with the repeat course started after a 4-week interval. During the treatment, if side effects greater than WHO grade 2 were observed, further treatment was postponed for up to 4 weeks. If side effects persisted for more than 4 weeks, treatment was discontinued. No dose modification was allowed. If disease progression was observed during the chemotherapy, patients were discontinued from the study.

After completion of the chemotherapy, patients were reassessed for resectability on the basis of their general condition and extent of disease, including assessment by diagnostic imaging. Laparotomy was indicated only if a radical excision was considered to be possible. The radical procedure performed was, basically, total gastrectomy with lymph node dissection (D2), including para-aortic lymph node dissection.

\section{Evaluation of response and toxicity}

In measurable metastatic lesions, standard WHO criteria were adopted for the response evaluation. The response of primary foci was evaluated by the criteria of the Japanese Research Society of Gastric Cancer. Because this study targeted scirrhous cancer, responses for primary foci were evaluated as c-lesions (i.e., macroscopically diffuse type). In the primary foci, evaluation was based on the recovery of the elasticity of the gastric wall, which was determined on a barium-filled X-ray film taken with the patient in the upright position. Complete response (CR) was defined as disappearance of all invasive findings and partial response (PR) as more than $50 \%$ decrease of the affected area on the X-ray film taken in the same position as that before treatment. Progressive disease (PD) was defined as an increase in lesions by $25 \%$, or the appearance of new lesions. The remaining cases were classified as stable disease (SD). WHO toxicity criteria were adopted for determining the toxicity of the chemotherapy.

Pathological assessment was used to evaluate disease extent, resection margins, and evidence of necrotic, as well as viable, tumor. The pathological responses of the specimen were classified according to the criteria presented by the Japanese Research Society for Gastric 
Cancer: grade 0 , no necrosis or degeneration owing to the chemotherapy is observed; grade $1 \mathrm{a}$, necrosis or degeneration owing to the chemotherapy is observed in less than one-third of the tumor; grade $1 \mathrm{~b}$, less than twothirds and more than one-third of the tumor show such an effect; grade 2, more than two-thirds of the tumor shows such an effect; grade 3, all of the tumor shows such an effect.

\section{Statistical analysis}

The primary endpoint of this study was the 2-year survival rate. Forty-five patients were required to be registered, on the basis that the 2-year survival rate of patients receiving this neoadjuvant therapy was expected to be $50 \%$, allowing for $10 \%$ of ineligible patients. When 20 patients were registered in this study, an interim analysis was performed in order to determine whether to continue further accrual.

Median survival time was calculated from the initial date of the initiation of the chemotherapy to the date of death or the last follow-up date. Median time to progression was calculated from the initial date of the initiation of the chemotherapy to the date documented for any progressive disease, the date of death, or the last follow-up date. These survival data were analyzed according to the method of Kaplan and Meier, and compared with the data for historical controls (371 patients with scirrhous cancer, treated initially with surgery at the National Cancer Center Hospital, between 1980 and 1990).

\section{Results}

\section{Patient characteristics}

Between June 1994 and September 1997, a total of 20 patients were enrolled in the study. Because the response rate in this group was lower than expected, further accruals were discontinued, and survival was evaluated in these 20 patients. Analysis, in September 1999 , showed that the 2-year survival rate of the 20 patients was lower than the expected rate, and the study was closed. The characteristics of the 20 patients are summarized in Table 1 . Their median age was 54 years, with a range of 38 to 67 years. All patients had a pretreatment ECOG performance status of 0 or 1 , and in 19 of the $20(95 \%)$ patients, the histology of their primary tumor was undifferentiated type.

\section{Response to chemotherapy}

Seventeen $(85 \%)$ of the 20 patients completed two courses of preoperative FAMTX therapy. There were 2
Table 1. Patient characteristics

\begin{tabular}{lc} 
No. of patients & 20 \\
Median age, in years (range) & $54(38-67)$ \\
Male/female & $11 / 9$ \\
Pretreatment ECOG performance status & \\
$\quad 0-1$ & \\
$\geqq 2$ & 0 \\
Histologic type & \\
$\quad$ Differentiated & 1 \\
$\quad$ Undifferentiated & 19 \\
\hline ECOG, Eastern Cooperative Oncology Group &
\end{tabular}

Table 2. Toxicity of FAMTX

\begin{tabular}{lrrrrr}
\hline & \multicolumn{5}{c}{ No. of patients by grade } \\
\cline { 2 - 6 } Toxicity & 0 & 1 & 2 & 3 & 4 \\
\hline WBC & 0 & 1 & 5 & 6 & 8 \\
Neutrophil & 0 & 2 & 4 & 8 & 6 \\
PLT & 14 & 1 & 3 & 2 & 0 \\
Nausea/vomiting & 4 & 11 & 3 & 2 & 0 \\
Diarrhea & 14 & 2 & 1 & 2 & 1 \\
Stomatitis & 11 & 4 & 4 & 0 & 1 \\
Infection & 18 & 0 & 0 & 2 & 0 \\
Allergic reaction & 20 & 0 & 0 & 0 & 0 \\
Alopecia & 18 & 1 & 1 & 0 & 0 \\
Neurosensory & 19 & 0 & 0 & 1 & 0 \\
Neuromotor & 20 & 0 & 0 & 0 & 0 \\
Renal failure & 20 & 0 & 0 & 0 & 0 \\
Dyspnea & 20 & 0 & 0 & 0 & 0 \\
Hand-foot syndrome & 17 & 1 & 1 & 1 & 0 \\
\hline WBC, White blod & & &
\end{tabular}

WBC, White blood cell; PLT, platelet; FAMTX, 5-Fluorouracil plus Adriamycin plus methotrexate

patients with progressive disease (PD) after one course of FAMTX, and they underwent surgery immediately. One patient did not receive the first dose of Adriamycin because of grade 3 diarrhea caused by day 1 MTX and 5-FU, and directly underwent surgery. The results for response to chemotherapy in the 20 patients were as follows: no CR, 3 PRs, 14 SDs, and 3 PDs. The overall response rate was $15 \%$.

\section{Toxicity}

Myelosuppression was the major toxicity of the FAMTX regimen (Table 2). The median neutrophil count at the nadir was $1653 / \mu l$. Grade 3 or 4 neutropenia was observed in 14 of the 20 patients, 11 of whom required granulocyte-colony-stimulating factor (G-CSF) support. Administration of Adriamycin on day 15 was postponed in 12 patients because of severe neutropenia. Grade 3 or 4 diarrhea was observed in 3 patients (15\%). Grade 3 hand-foot syndrome and pneumonia were observed in only 1 patient each. No death caused by drug toxicity was observed in this cohort of 20 patients. 


\section{Surgical outcome}

Of the 20 patients, 18 underwent gastrectomy, including 13 curative resections with negative histological margins. The remaining 2 patients were classified as having PD and SD, respectively, after the neoadjuvant chemotherapy, as they had unresectable tumors at operation owing to severe peritoneal seeding and direct invasion of adjacent organs; probe laparotomy was therefore performed.

Peritoneal washing cytology before chemotherapy was performed in 16 patients. In these 16 patients, 3 were proven to be positive for cancer cells. Two of the 3 patients who had been shown to be positive for cancer cells in preoperative peritoneal washing cytology showed severe peritoneal seeding, while only 2 of the 13 patients who were negative for cancer cells had peritoneal dissemination at laparotomy.

The mean operative time was $5 \mathrm{~h}$, and the mean volume of intraoperative blood loss was $969 \mathrm{ml}$. As surgical complications, two patients developed minor pancreatic fistula and one patient developed stenosis of the esophago-jejuno anastomosis. There was no mortality caused by the surgical procedures. No significant difference was observed with regard to operative time, intraoperative blood loss, and morbidity rate between the patients in this study and the historical controls (those with scirrhous gastric cancer who had received gastrectomy without neoadjuvant chemotherapy at our institution; data not shown).

\section{Histological outcome}

Eighteen resected specimens were examined for the histological effects of the chemotherapy (Table 3). No grade 2 or 3 histological effects were observed in these 18 resected specimens.

\section{Survival}

Four of the 20 patients $(20 \%)$ were still alive at a median follow up of 59 months: 2 patients had recurrent disease and 2 had no evidence of disease. Sixteen patients had developed peritoneal dissemination and had already died of the recurrent disease. Figure 1 shows the survival curve of the 20 patients. The median survival time was 16.4 months, and the 2-year actual survival rate was $25 \%$. The 2 -year actuarial survival of the historical controls was $27 \%$, and there was no difference in 2-year survival between the present patients and the historical controls.

\section{Discussion}

The prognosis of scirrhous cancer is still far worse than that of nonscirrhous gastric cancer. One of the major reasons for the poorer prognosis of scirrhous cancer is that, at the time of diagnosis, most patients have potentially unresectable disease, in particular, with peritoneal dissemination. Neoadjuvant chemotherapy in scirrhous gastric cancer is a logical approach to improving surgical resectability and reducing the incidence of subsequent distant metastatic disease.

Several studies of neoadjuvant chemotherapy for scirrhous cancer have been reported [14-17]. Eriguchi

Table 3. Pathological outcome

\begin{tabular}{ll} 
Grade III & 0 \\
Grade II & 0 \\
Grade Ib & 5 \\
Grade Ia & 9 \\
Grade 0 & 4 \\
\hline
\end{tabular}

Pathological responses of the specimens were classified according to the criteria of the Japanese Research Society for Gastric Cancer

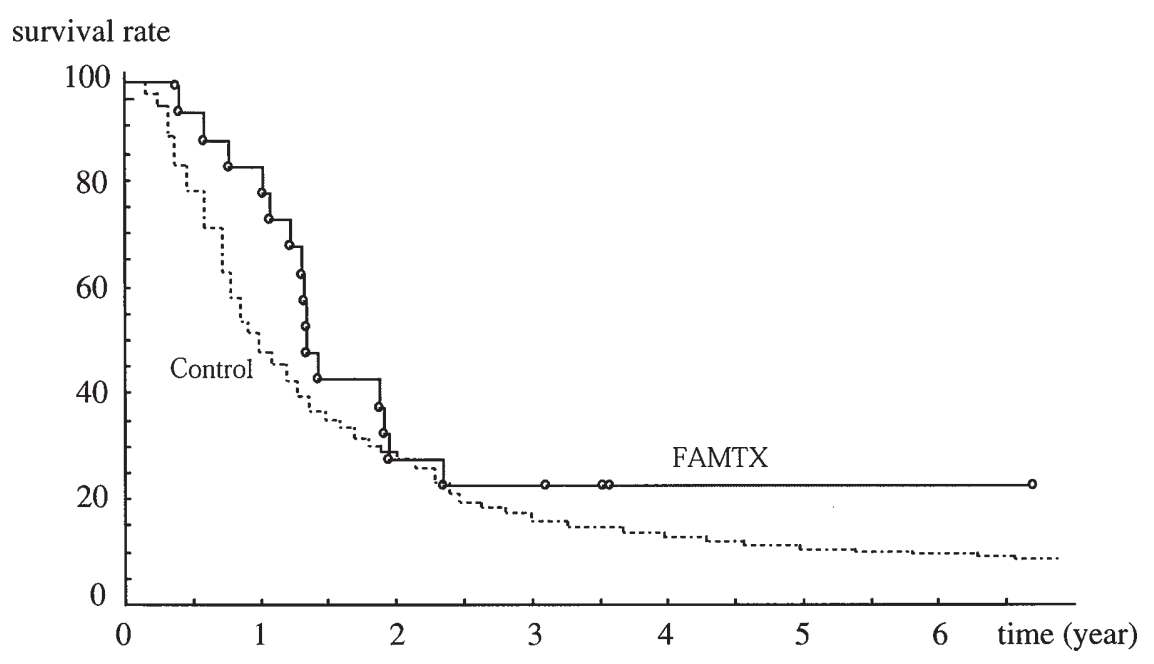

survival rate

Fig. 1. Cumulative survival curve, by Kaplan-Meier analysis, for 20 patients with scirrhous gastric cancer treated with 5-fluorouracil plus Adriamycin plus methotrexate $(F A M T X)$ as a neoadjuvant chemotherapy. There was no significant difference between the survival of the 20 patients in this study and that of the historical controls, as determined by a logrank test 
et al. [16] reported a pilot study of the preoperative administration of oxalliplatin (1-OHP) to 5 patients with scirrhous gastric cancer; there were three grade 2 and two grade 1a histological responses according to the criteria of the Japanese Research Society for Gastric Cancer. Suga et al. [17] also described a neoadjuvant chemotherapy, using uracil and tegafur plus cisplatin, in patients with potentially unresectable scirrhous gastric cancer. Ascites from peritoneal dissemination disappeared in 8 of the 13 patients $(62 \%)$ who had received uracil, and 10 of the total number of 28 patients in the trial underwent surgical resections after the preoperative regimen. However, these studies were small and the results could not be used to determine whether these treatments were indicated for potentially unresectable or resectable disease. Thus, in the absence of other relevant trials, the efficacy of neoadjuvant chemotherapy for scirrhous gastric cancer remains to be established.

In our present study, the FAMTX regimen was feasible, despite substantial myelosuppression. No severe complication was observed during the surgical procedures. In 13 of the 20 patients, curative resection was achieved, and this appeared to be a higher curative resection rate than that in the historical controls. However, the patient response rate of $15 \%$ to the chemotherapy was disappointing, and the pathological response in the resected specimens were dismal. The 2year survival rate of the 20 enrolled patients was $25 \%$, which was lower than expected. The data from the historical controls treated at the National Cancer Center Hospital between 1980 and 1990, show that the 2-year survival of patients with scirrhous cancer treated with surgery as an initial therapy was $27 \%$. There were no differences in 2-year survival between the present patients and the historical controls. Most of our present patients developed peritoneal metastasis, despite the higher curative resection rate. These results suggest that FAMTX does not have sufficient efficacy for treating micrometastasis in the peritoneum.

Several randomized trials comparing FAMTX with other regimens appeared after we began the present study. Webb et al. [18] reported a randomized study that compared a combination of epirubicin, CDDP, and protracted infusion of 5-FU (ECF) with FAMTX. The median survival duration in patients with ECF (8.9 months) was significantly longer than that in the patients with FAMTX (5.7 months), and the global quality-of-life scores were better for ECF than for FAMTX. A report from the EORTC of a randomized phase III trial between FAMTX, etoposide + leucovorin + bolus 5-FU (ELF), and FP has been published by Vanhoefer et al. [19]. Each regimen demonstrated modest clinical efficacy, but there were no significant differences between the three regimens with regard to survival. Vanhoefer et al. [19] concluded that none of these investigated regimens should be regarded as the standard regimen for advanced gastric cancer. Therefore, the use of FAMTX has declined in recent years, and the development and investigation of new effective agents is necessary.

In our study, preoperative evaluation of peritoneal seeding that was not detectable by medical imaging was performed, using peritoneal washing cytology of the Douglas pouch, performed with the patient under local anesthesia. The procedure was very convenient, but it may be inferior to laparoscopic examination, both in terms of sensitivity, and in terms of stress to the patient. Therefore, in similar studies of neoadjuvant chemotherapy, in future, laparoscopic examination may be preferable for evaluating preoperative peritoneal dissemination.

In summary, neoadjuvant FAMTX followed by radical surgery was feasible in patients with potentially resectable scirrhous gastric cancer, but the responses, both clinically and histologically, were disappointing. Although the curative resection rate was improved by FAMTX, no survival advantage was observed.

\section{References}

1. Koizumi W, Kurihara M, Sasai T, Yoshida S, Morise K, Imamura A, et al. A phase II study of combination therapy with 5'-deoxy5 -fluorouridine and cisplatin in the treatment of advanced gastric cancer with primary foci. Cancer 1993;72:658-62.

2. Shimada Y, Yoshida S, Ohtsu A, Seki S, Shimizu S, Saito H, et al. A phase II study of EAP (etoposide, adriamycin and cisplatin) in the patients with advanced gastric cancer: Multiinstitutional study. J Jpn Soc Cancer Ther 1991;26:280.

3. Ohtsu A, Shimada Y, Yoshida S, Saito H, Seki S, Morise K, et al. Phase II study of protracted infusional 5-fluorouracil combined with cisplatinum for advanced gastric cancer: Report from the Japan Clinical Oncology Group (JCOG). Eur J Cancer 1994;30A: 2091-2093.

4. Lerner A, Gonin R, Steele GD Jr, Mayer RJ. Etoposide, doxorubicin, and cisplatin chemotherapy for advanced gastric adenocarcinoma: results of a phase II trial. J Clin Oncol 1992;10:536-40.

5. O'Connell MJ. Etoposide, doxorubicin, and cisplatin chemotherapy for advanced gastric cancer: an old lesson revisited. J Clin Oncol 1992;10:515-6.

6. Klein HO, Wickramanayake PD, Dieterle F, Mohr R, Oerkermann H, Brock J, et al. Chemotherapy schedule for the management of metastasizing gastric cancer. Methotrexate, adriamycin and 5-fluorouracil. Dtsch Med Wochenschr 1982;107:1708-12.

7. Wils J, Bleiberg H, Dalesio O, Blijham G, Mulder N, Planting A, et al. An EORTC Gastrointestinal Group evaluation of the combination of sequential methotrexate and 5-fluorouracil, combined with adriamycin in advanced measurable gastric cancer. J Clin Oncol 1986;4:1799-803.

8. Wils JA, Klein HO, Wagener DJ, Bleiberg H, Reis H, Korsten F, et al. Sequential high-dose methotrexate and fluorouracil combined with doxorubicin - a step ahead in the treatment of advanced gastric cancer: a trial of the European Organization for Research and Treatment of Cancer Gastrointestinal Tract Cooperative Group. J Clin Oncol 1991;9:827-31.

9. Kelsen D, Atiq OT, Saltz L, Niedzwiecki D, Ginn D, et al. FAMTX versus etoposide, doxorubicin, and cisplatin: a 
random assignment trial in gastric cancer. J Clin Oncol 1992;10: 541-8.

10. Murad AM, Santiago FF, Petroianu A, Rocha PR, Rodrigues MA, Rausch M. Modified therapy with 5-fluorouracil, doxorubicin, and methotrexate in advanced gastric cancer. Cancer 1993;72: $37-41$.

11. Kelsen D, Karpeh M, Schwartz G, Gerdes H, Lightdale C, Botet $\mathrm{J}$, et al. Neoadjuvant therapy of high-risk gastric cancer: a phase II trial of preoperative FAMTX and postoperative intraperitoneal fluorouracil-cisplatin plus intravenous fluorouracil. J Clin Oncol 1996;14:1818-28.

12. Songun I, Keizer HJ, Hermans J, Klementschitsch P, de Vries JE, Wils JA, et al. Chemotherapy for operable gastric cancer: results of the Dutch randomised FAMTX trial. The Dutch Gastric Cancer Group (DGCG). Eur J Cancer 1999;35:558-62.

13. Westermann AM, Taal BG, Swart M, Boot H, Craanen M, Gerritsen WR. Sequence-dependent toxicity profile in modified FAMTX (fluorouracil-adriamycin-methotrexate) chemotherapy with lenograstim support for advanced gastric cancer: a feasibility study. Pharmacol Res 2000;42:151-6.

14. Mai M, Ogino T, Ueda H, Ooi A, Takahashi Y, Sawaguchi K, et al. Study on neoadjuvant chemotherapy of Borrmann 4 type carcinoma of stomach and its clinical significance. Nippon Gan Chiryo Gakkai Shi (J Jpn Soc Cancer Ther) 1990;25:586-97.
15. Maeda O, Iwase H, Mamiya N, Nakamura M, Mizuno T, Nishio $\mathrm{Y}$, et al. Scirrhous cancer of the stomach which survived for more than 5 years after neoadjuvant chemotherapy with UFT (uracil and tegafur) and cisplatin. Intern Med 2000;39:239-44.

16. Eriguchi M, Osada I, Fujii Y, Takeda Y, Yoshizaki I, Akiyama N, et al. Pilot study for preoperative administration of 1-OHP to patients with advanced scirrhous type gastric cancer. Biomed Pharmacother 1997;51:217-20.

17. Suga S, Iwase H, Shimada M, Nishio Y, Ichihara T, Ichihara S, et al. Neoadjuvant chemotherapy in scirrhous cancer of the stomach using uracil and tegafur and cisplatin. Intern Med 1996;35:9306.

18. Webb A, Cunningham D, Scarffe JH, Harper P, Norman A, Joffe $\mathrm{JK}$, et al. Randomized trial comparing epirubicin, cisplatin, and fluorouracil versus fluorouracil, doxorubicin, and methotrexate in advanced esophagogastric cancer. J Clin Oncol 1997;15:261-7.

19. Vanhoefer U, Rougier P, Wilke H, Ducreux MP, Lacave AJ, Van Cutsem E, et al. Final results of a randomized phase III trial of sequential high-dose methotrexate, fluorouracil, and doxorubicin versus etoposide, leucovorin, and fluorouracil versus infusional fluorouracil and cisplatin in advanced gastric cancer: a trial of the European Organization for Research and Treatment of Cancer Gastrointestinal Tract Cancer Cooperative Group. J Clin Oncol 2000;18:2648-57. 